Revue de la littérature

\title{
Neurones miroirs, substrat neuronal de la compréhension de l'action?
}

\section{Mirror neurons, neural substrate of action understanding?}

\author{
D. Zarka ${ }^{\mathrm{a}, \mathrm{b}, *}$, A.M. Cebolla ${ }^{\mathrm{a}}, \mathrm{G}$. Cheron $^{\mathrm{a}, \mathrm{c}}$ \\ a Faculté des Sciences de la Motricité, laboratoire de neurophysiologie et de biomécanique du mouvement, université Libre de Bruxelles, CP640, 808, route \\ de Lennik, 1070 Brussels, Belgique \\ ${ }^{\mathrm{b}}$ Unité de Recherche en Sciences de l'Ostéopathie, faculté des Sciences de la Motricité, université Libre de Bruxelles, CP640, 808, route de Lennik, 1070 \\ Brussels, Belgique \\ c Laboratoire d'électrophysiologie, université de Mons, 7000 Mons, Belgique
}

\section{N F O A A R T I C L E}

\section{Historique de l'article :}

Reçu le 26 mars 2021

Accepté le 12 juin 2021

Disponible sur Internet le xxx

\section{Mots clés :}

Neurones miroirs

Compréhension de l'action

Singe

Rythme mu

\section{R É S U M É}

En 1992, le Laboratoire de physiologie humaine de l'université de Parme (Italie) publie une étude décrivant, chez le macaque, des neurones " miroirs " s'activant à la fois lorsque le singe réalise une action et lorsqu'il observe un expérimentateur effectuer cette même action. L'équipe de recherche à l'origine de cette découverte postule que le système des neurones miroirs est la base neurale de notre capacité à comprendre l'action d'autrui au travers d'une cartographie motrice de l'action observée sur le répertoire moteur de l'observateur (direct-matching hypothesis). Cette conception rencontre néanmoins de sérieuses critiques. Ces critiques tentent de relativiser la fonction des neurones miroirs en les replaçant au sein d'un maillage d'interdépendances neurocognitives et sensorielles. En somme, la caractéristique essentielle de ces neurones est d'associer le traitement de l'information sensorielle, notamment visuelle, avec celui de l'information motrice. Leur fonction élémentaire serait ainsi de fournir une simulation motrice de l'action observée, à partir des informations visuelles de celle-ci. Ils peuvent contribuer, de concert avec d'autres aires cérébrales non-miroirs, à l'identification/prédiction du but de l'action et à l'interprétation de l'intention de l'acteur qui l'effectue. L'étude de la connectivité et des synchronisations à haute fréquences des différentes aires cérébrales en jeu lors de l'observation d'une action apporterait probablement des informations importantes quant à la contribution dynamique des neurones miroirs à la « compréhension » de l'action d'autrui. La présente revue a pour vocation d'offrir au lecteur une analyse actualisée des preuves scientifiques relatives aux neurones miroirs et de leur fonction élémentaire, ainsi que de l'éclairer quant à leur contribution à notre faculté d'interpréter et de comprendre l'action d'autrui. (c) 2021 L'Encéphale, Paris.

\section{A B S T R A C T}

In 1992, the Laboratory of Human Physiology at the University of Parma (Italy) publish a study describing "mirror" neurons in the macaque that activate both when the monkey performs an action and when it observes an experimenter performing the same action. The research team behind this discovery postulates that the mirror neurons system is the neural basis of our ability to understand the actions of others, through the motor mapping of the observed action on the observer's motor repertory (direct-matching hypothesis). Nevertheless, this conception met serious criticism. These critics attempt to relativize their function by placing them within a network of neurocognitive and sensory interdependencies. In short, the essential characteristic of these neurons is to combine the processing of sensory information, especially visual, with that of motor information. Their elementary function would be to provide a motor simulation

\footnotetext{
* Auteur correspondant.

Adresse e-mail : david.zarka@ulb.be (D. Zarka).
} 
of the observed action, based on visual information from it. They can contribute, with other non-mirror areas, to the identification/prediction of the action goal and to the interpretation of the intention of the actor performing it. Studying the connectivity and high frequency synchronizations of the different brain areas involved in action observation would likely provide important information about the dynamic contribution of mirror neurons to "action understanding". The aim of this review is to provide an up-todate analysis of the scientific evidence related to mirror neurons and their elementary functions, as well as to shed light on the contribution of these neurons to our ability to interpret and understand others' actions.

(c) 2021 L'Encéphale, Paris.

\section{Introduction}

En 1992, le Laboratoire de physiologie humaine de l'université de Parme présente une étude décrivant, chez le macaque, des neurones s'activant à la fois lorsque le singe réalise une action et lorsqu'il observe un expérimentateur effectuer cette même action. Cette étude fut publiée dans Experimental Brain Research et passa relativement inaperçue [1]. En 1996, cette équipe, dirigée par Rizzolatti, publie deux nouvelles études [2,3] où apparut pour la première fois le terme de «neurones miroirs ». Sur la base de cette découverte, ces deux publications proposent un modèle physiologique capable de transcrire un pattern visuel d'une action en une compréhension de celle-ci, par la simple correspondance de l'action observée sur le répertoire moteur de l'observateur (direct-matching hypothesis, [4]). Ce modèle " miroir " fut rapidement étendu à plusieurs phénomènes cognitifs humains (imitation, empathie et langage) et décliné comme une base neurophysiologique de la compréhension de l'autre au sens large.

Aujourd'hui, le paradigme miroir est largement dominant dans le domaine des neurosciences cognitives, et la fonction des neurones miroirs est sujette à de multiples spéculations. Cet engouement est probablement dû au fait que ce modèle concerne symboliquement le dualisme entre le soi et l'autre, et représente une porte ouverte à l'étude neurophysiologique de sujets originellement réservés à la psychologie, à la sociologie, voire à la philosophie. Cependant, le noyau dur du modèle selon lequel le système miroir serait à l'origine de notre compréhension de l'action, reste source de nombreux débats. La présente revue a pour vocation d'offrir au lecteur une analyse actualisée des preuves scientifiques relatives aux neurones miroirs et de leurs fonctions élémentaires, en particulier de leur contribution à l'interprétation et à la compréhension de l'action d'autrui.

\section{Les neurones miroirs chez le singe}

\subsection{Spécificités visuomotrices des neurones miroirs}

Découverts initialement dans l'aire F5 et dans le lobe pariétal inférieur (IPL) du macaque, les neurones miroirs sont une classe particulière de cellules visuomotrices. Leur spécificité est de décharger à la fois lorsque le singe effectue une action ${ }^{1}$ (par exemple, " saisir un objet ") mais également lorsque, au repos, il observe un semblable, singe ou humain, effectuer cette même

\footnotetext{
${ }^{1}$ Le terme " action » désigne un mouvement qui possède une valeur sémantique, c'est-à-dire ayant un but ou un sens en dehors de l'exécution du mouvement luimême. Nous pouvons distinguer deux types d'action : une action peut être engagée vis-à-vis d'un ou de plusieurs objets (ex. saisir un objet, remplir un verre d'eau). Elle sera alors qualifiée de transitive. Autrement, elle peut être purement communicative (ex. pointer du doigt, tirer la langue). Elle sera alors qualifiée d'intransitive. Par opposition, un mouvement ne possédant pas de valeur sémantique n'a pas de sens pour l'observateur. À des fins de simplicité, nous emploierons le mot " geste " pour désigner indifféremment un mouvement avec ou sans valeur sémantique.
}

action [1,5-7]. Contrairement aux neurones moteurs dit " canoniques $\|^{2}$, les neurones miroirs ne répondent pas à la seule présentation d'objets, pas même à la présentation d'objets ayant une certaine valeur d'intérêt telle que de la nourriture. Leur activité ne semble pas modulée par la répétition de l'action observée $[9,10]$. À l'exécution comme à l'observation, leur réponse débute au commencement du mouvement et s'arrête à la fin [2]. De plus, les neurones miroirs chez le singe ne sont stimulés que par des actions, c'est-à-dire, des gestes possédant une certaine valeur sémantique, transitifs ou intransitifs $[2,3,11]$.

Plusieurs études tendent à montrer en outre, que l'activité des neurones miroirs ne se limite pas à la description cinématique de l'action, mais qu'elle dépendrait du but et de l'intention sous-jacente de l'action observée. Rochat et al. [12] montrent par exemple que les neurones miroirs de F5 déchargent, quel que soit le mouvement nécessaire pour la réaliser, l'action « saisir » (à la main, avec une pince). En outre, cette activation serait également enregistrée dans le cas d'un outil (une pince inversée) qui n'aurait jamais été utilisé par le singe, ce qui suggère qu'elle serait liée au résultat effectif ( " but ») de l'action [12]. L'étude de Umiltà et al. [13] montre que les neurones miroirs s'activent également pour des actions (" saisir ") partiellement cachées (par un écran) pour autant que le singe ait les éléments nécessaires à la déduction du but de l'action (présentation préalable de l'objet à saisir, et placé derrière l'écran). En outre, Kohler et al. [14] comparent, chez le singe, la réponse de neurones miroirs de $\mathrm{F} 5$ à des actions discriminables auditivement (ex : « casser une cacahuète ", " déchirer du papier »). Ils démontrent que certains neurones miroirs déchargent tant à l'exécution et à l'observation, qu'au bruit de ces actions [14]. Une partie de ces neurones démontre une préférence pour l'une des actions, sans différence significative entre les conditions d'observation ou d'écoute [15].

Les actions s'effectuant rarement de façon isolée, Fogassi et al. [16] se sont intéressés à l'activité des neurones miroirs dans le cadre d'actions complexes formées de plusieurs actions. L'action " saisir " peut en effet, être suivie de " amener à la bouche " (pour former l'action " manger ") ou de " placer dans un récipient " (pour former l'action " ranger "). Les auteurs démontrent que lors de la phase " saisir " (c'est-à-dire, au moment du contact entre la main et l'objet, et donc avant le début de la deuxième partie du mouvement) la majorité des neurones miroirs de l'IPL répondent à l'exécution et à l'observation en fonction de l'action qui suit ( " manger » ou " ranger »). Les auteurs y précisent néanmoins que la présence ou l'absence du récipient indique clairement l'action que l'expérimentateur s'attend à réaliser [16]. Dans la même veine, une étude récente de Coudé et al. [17] démontre chez le singe que lors de l'observation d'une action (" saisir ") la réponse des neu-

\footnotetext{
2 Les " neurones canoniques " répondent à la présentation d'un objet seul. L'activité de ces derniers est généralement associée à l'affordance d'un objet, c'està-dire à la capacité de l'objet à suggérer sa propre utilisation. Il existe également des neurones aux propriétés doubles : canonique et miroir [8].
} 
rones miroirs du cortex prémoteur ventral (PMv) est influencée par l'orientation du regard de l'acteur (congruent ou non avec la tâche réalisée). Ces résultats suggèrent ainsi que la représentation de l'action observée s'élabore non seulement à partir des informations cinématiques du mouvement mais également sur des informations contextuelles à valeur sociale [17].

\subsection{Topographie et réseau des neurones miroirs}

L'études des neurones miroirs chez le singe démontrent ainsi que plusieurs régions du cerveau contiennent des neurones miroirs et que celles-ci s'organisent en système. Dès lors, le traitement visuomoteur de l'information prendrait la forme d'un ensemble fonctionnellement organisé de neurones : un réseau de neurones miroirs (RMN). Ce réseau inclut en particulier la partie ventrale du cortex prémoteur (PMv) du singe macaque (appelée aire F5 [1-3]) et l'IPL [16]. Au sein de l'IPL, ce sont plus précisément les aires PF/PFG et l'aire intrapariétale antérieure (AIP) qui présenteraient une importante activité miroir $[16,18,19]$. Les aires F5 et PFG seraient liées respectivement à l'encodage du but immédiat de l'action et de l'intention motrice [20]. Dans ce cadre, les neurones de l'aire F6 (pré-SMA) intègreraient les informations relatives à l'objet et au contexte, et génèreraient un signal relatif à la réalisation d'un objectif pendant la réalisation de l'action [21]. Les neurones de l'AIP coderaient le point de vue de l'observation (latérale, frontale), la posture de l'acteur (debout, assis), et participeraient à l'identification de l'action observée [19]. Il a été également montré que l'aire motrice primaire F1 (M1) et la partie dorsale du cortex prémoteur (PMd) du singe présentent des propriétés miroirs dans le cadre de tâches complexes [6,22]. Une étude par injection d'un traceur chez le singe montre en outre que PMv et l'IPL partagent des connections avec de nombreuses structures corticales (PMd, M1, cortex somatosensoriel secondaire, cortex préfrontal ventrolatéral et l'insula) mais également avec des structures sous-corticales telles que le thalamus et le claustrum [23].

Par ailleurs, bien qu'aucune étude n'ait pu attester l'existence de neurones miroirs dans le sulcus temporal supérieur (STS), cette région serait étroitement liée à l'activité des neurones miroirs. À ce titre, des projections bidirectionnelles de PF vers l'aire F5 et le STS ont été mises en évidence, suggérant une complicité fonctionnelle de ces trois régions [24,25]. Des études fMRI lors de l'observation d'une action (" saisir ") chez le singe montrent également que les parties ventrale et dorsale du STS se projettent vers PFG et AIP respectivement, et que ces deux aires se projettent elles-mêmes sur différentes parties de F5 [26,27].

\subsection{Paramètres modulant l'activité du RNM}

Au même titre que les neurones moteurs sont sensibles à de nombreux paramètres du mouvement, l'activité miroir varierait en fonction de la cinématique du mouvement [11], le caractère transitif ou intransitif de l'action exécutée/observée et de l'effecteur de premier plan qui réalise l'action, c'est-à-dire la main droite ou gauche, la bouche, un outil, etc. [2,5,12]. Elle est également influencés par l'association d'une valeur subjective (associée par exemple à une récompense) de l'objet en jeu [28]. De plus, l'activité miroir change en fonction de la distance et de la capacité de l'observateur à interagir vis-à-vis de l'action observée [29]. Certains neurones miroirs déchargent préférentiellement pour une action distante (extrapersonnal space) de l'observateur quand d'autres déchargent spécifiquement pour une action proche de celui-ci (peripersonal space). À une distance peripersonnelle égale, certains neurones cessent de décharger si l'action est exécutée en dehors du champ d'action de l'observateur (séparé par une vitre) par rapport à une action à sa portée [30]. Ils sont en outre sensible au mode d'affichage de l'action - naturelle ou vidéo - et au point de vue de l'observation - à la 1 ère personne, de profil ou de face [31].

Différentes études suggèrent également que les neurones miroirs sont sous l'influence de différents mécanismes d'inhibition, de manière à éviter toute exécution motrice lors de l'observation. Rizzolatti et al. [32] suggèrent notamment que l'aire F6 (pré-SMA) serait à l'origine d'une modification fonctionnelle de F5 en fonction des conditions d'exécution ou d'observation, passant d'une excitation motrice dans le premier cas à son inhibition dans le second. D'après Kraskov et al. [33,34] le système miroir possèderait un mécanisme d'inhibition motrice intrinsèque. Les auteurs ont, en effet, mis en évidence une activité miroir au niveau de neurones pyramidaux (PTN) de F5. Parmi les neurones déchargeant à l'exécution de l'action " saisir ", certains montraient notamment une suppression complète de leurs activités lors de l'observation de celle-ci [33]. Une étude similaire [35] aux niveau de F1 a également montré que certains PTN sont facilitateurs du mouvement, augmentant leur fréquence de décharge tant à l'exécution qu'à l'observation de l'action. D'autres néanmoins diminuent leur fréquence de décharge à l'observation spécifiquement. Ils auraient ainsi un rôle d'inhibition du mouvement lors de l'observation, qui expliquerait l'absence d'exécution motrice au cours de l'observation [34]. Une récente étude de Jerjian et al. [36] s'est intéressée à l'activité des PTN de F1 et F5 chez deux singes durant l'exécution, l'observation et l'inhibition (NoGO) d'une action. Celle-ci révèle que certains PTN de F1 modulent leur activité en fonction de la condition exécution ou observation. En outre, certains PTN de F1 présentent un pattern d'activité lors de l'observation proche de celui constaté lors de la condition d'inhibition. Ces derniers neurones agiraient probablement sur des interneurones médullaires inhibiteurs de l'action [36].

\section{Le réseau de neurones miroirs chez l'homme}

\subsection{Signe d'un réseau de neurones miroirs chez l'homme : le rythme mu}

Les premières études appuyant sans le savoir l'existence d'un système miroir chez l'homme datent des années 1950. Elles démontrent en effet, à l'aide de l'EEG, que l'observation de mouvements désynchronise les rythmes mu $(8-13 \mathrm{~Hz})$ présents au repos [37]. Or, cette désynchronisation est décrite en particulier au niveau central lors de tâches motrices et reflètent l'activité de neurones moteurs et pré-moteurs qui y est sous-jacente. C'est ainsi que, suite aux premières publications introduisant la notion de neurones miroirs, l'analogie entre des désynchronisations du rythme mu lors de l'exécution et lors de l'observation d'une même tâche motrice fut interprétée comme le reflet d'une activité des neurones miroirs [38]. Cette désynchronisation lors des deux conditions fut reproduite et discutée à de multiples reprises [39-43], voir [44] pour une meta-analyse]. Cette interprétation a également été corroborée par l'utilisation conjointe de l'EEG et de la fMRI, indiquant que la désynchronisation du rythme mu durant l'observation de l'action est corrélée à l'activité de certaines aires considérées comme miroirs chez l'homme, à savoir l'IPL, l'aire motrice supplémentaire (SMA) et PMd $[45,46]$. Ces études indiquent néanmoins que d'autres structures sensibles notamment aux informations visuelles, sont également impliquées dans la suppression du rythme mu. Elles soulignent ainsi la difficulté d'associer celle-ci à la seule activité des neurones miroirs $[45,46]$.

Un certain nombre de données suggèrent cependant que l'activité des neurones miroirs serait prépondérante dans la suppression du rythme $\mu$ lors de l'observation. Urgen et al. [47] ont par exemple montré que le rythme mu (contrairement au rythme thêta) n'était pas sensible à la seule apparence biologique 
ou mécanique de l'action observée (réalisée par un humain, un androïde ou un robot). En outre, une étude EEG récente chez l'enfant de 9 mois démontre que les régions centro-occipitales sont fonctionnellement plus connectées que les autres régions lors de la désynchronisation du rythme mu à l'observation des mouvements [48]. De plus, à l'instar des données sur le singe, la suppression du rythme mu est non seulement corrélée à l'augmentation des réponses musculaires évoquées par stimulation transcrânienne (TMS) lors de l'observation d'un mouvement de la main, mais également influencée par l'orientation du regard de l'acteur [49]. De nombreuses études indiquent également que la désynchronisation du rythme mu dépendrait du répertoire moteur de l'observateur. Van Elk et al. [50] constate notamment une désynchronisation du rythme mu (et bêta) plus importante chez les bébés lors de l'observation d'un déplacement à quatre pattes que de la bipédie. La taille de l'effet semble en outre corrélée à l'expérience de l'enfant dans la marche à quatre pattes [50]. De même, plusieurs études [51,52] démontrent une augmentation de l'activité EEG des aires sensorimotrices lors de l'observation de mouvements complexes après un entraînement moteur pour ces mêmes mouvements. Or, cette augmentation n'apparaît pas dans le cadre d'entraînements reposant uniquement sur l'observation [51,52].

Cependant, l'étude de Coll et al. [53] indique que le rythme mu ne serait pas une mesure spécifique de l'activité des neurones miroirs, mais refléterait avant tout les aspects somatosensoriels de l'action observée/exécutée. Ainsi, la difficulté d'interpréter la suppression du rythme $\mu$ comme marqueur physiologique des neurones miroirs réside dans l'absence de preuves directes chez l'homme entre le recrutement des neurones miroirs et la variation de la puissance d'un rythme mesuré à la surface du cuir chevelu. À ce propos, un éclairage intéressant provient des expériences réalisées chez le singe par Bimbi et al. [54]. Ces auteurs ont enregistré simultanément les décharges neuronales (activité unitaire et multiple, MUA), les oscillations relatives aux potentiels de champs locaux (LFP) et l'EEG de surface au niveau de F5, durant l'exécution et l'observation d'une tâche de préhension. Leurs résultats démontrent que l'activité des différentes populations de neurones de PMv contribue à la génération et à la modulation du rythme mu. Ils montrent en effet, tant durant l'exécution que l'observation de l'action, une corrélation importante entre les MUA et l'augmentation de la puissance gamma des LFP. Or, cette augmentation gamma des LFP précède la désynchronisation des rythmes mu [54].

\subsection{Topographie du RNM chez l'homme}

Très tôt après la découverte des neurones miroirs chez le singe, plusieurs publications, portées par le développement des nouvelles techniques d'imagerie cérébrale, paraissent sur l'homme. Elles démontrent l'existence de zones d'activations communes dans les deux conditions [55-58], dont notamment une partie du cortex frontal inférieur (l'aire de Broca, BA44) considérée d'un point de vue phylogénétique comme l'homologue de l'aire F5 du singe [4]. Aujourd'hui, un grand nombre d'études confirment que l'observation et l'exécution d'actions activent plusieurs aires corticales communes. Une méta-analyse de Molenberghs et al. [59] met en évidence que le lobe pariétal inférieur [IPL ; BA40] et supérieur [SPL ; BA5/7], le cortex pré-moteur ventral [PMv ; BA6] et dorsal [PMd ; BA6] et le gyrus frontal inférieur [IFG ; BA44/45] sont les principales aires présentant une activité miroir. D'après les auteurs, elles formeraient le noyau du RNM chez l'homme [59].

Par ailleurs, Buccino et al. [60] démontrent que ces aires miroirs, frontales et pariétales, possèdent une organisation somatotopique. Ainsi, l'activité miroir de l'aire de Broca serait associée à l'observation de mouvements de la main et de la bouche ; l'activité du cortex précentral reflèterait des actions proximales du bras et du cou ; enfin la partie dorsale du gyrus précentral serait liée aux mouvements des jambes et des pieds [60]. Une autre étude de Jastorff et al. [61] s'intéresse à l'activité des aires pré-motrice et pariétale à l'observation de quatre type d'actions (" saisir ", " laisser tomber ", " tirer ", " pousser ») effectuées avec trois effecteurs différents (la bouche, la main et le pied). L'étude indique que les aires miroirs du cortex pré-moteur sont organisées en fonction de l'effecteur de l'action, alors que les aires pariétales sont organisées selon le type d'action observée. Elle montre en outre que l'IPL aurait une organisation actotopique, à savoir que : les actions consistant à ramener un objet à soi (" saisir », " tirer ») activent la partie ventrale de l'AIP (présumée de l'homme), alors que les actions consistant à éloigner de soi (" laisser tomber ", " pousser ") activerait la partie dorsale de cette même aire [61]. Simon et al. [62] montrent également que le RNM serait constitué de deux sous réseaux : l'un sensible aux niveaux de perception de l'action observée (incluant M1, PMd, STS), l'autre insensible à ceux-ci (incluant SMA, IFG, PMv, SPL).

Il importe néanmoins de noter que, contrairement aux enregistrements de potentiels d'actions de neurones isolés (single cell record [63]) chez le singe, les outils de mesures généralement employés chez l'homme (fMRI, PET, MEG, EEG, TMS) ne permettent à aucun moment d'affirmer avec certitude que les données obtenues sont le fruit de l'activité des mêmes neurones lors de l'observation et lors de l'exécution d'actions. La complexité des réseaux neuronaux et la résolution (temporelle ou spatiale) des techniques ne permettent de mettre en évidence que des corrélations d'activités à l'échelle d'aires cérébrales. À ce jour, une seule publication de Mukamel et al. [64] rapportent une preuve directe, sur la base de single cell records, de neurones aux propriétés miroirs chez l'homme au niveau de l'aire motrice supplémentaire et de l'hippocampe.

\subsection{Spécificité visuomotrice des neurones miroirs chez l'homme}

Comme nous l'avons vu, de nombreuses études chez le singe apportent des arguments sérieux en faveur d'un réseau organisé de neurones miroirs dont l'activité est en rapport étroit avec le but et l'intention de l'action observée. De même chez l'homme, un certain nombre d'études rapporte que l'activité des neurones miroirs est étroitement liée au but de l'action exécutée ou observée. L'étude de Buccino et al. [65] par exemple, analyse les activités corticales chez l'homme lors de l'observation par vidéo (sans son) d'actions orales transitives et intransitives réalisées par un humain (" mordre » et " parler "), un singe (" mordre " et " claquer des lèvres ") et un chien ( " mordre » et " aboyer »). Les résultats montrent que l'action " mordre » (quelle que soit l'espèce animale observée), " parler » et " claquer des lèvres » stimulent les aires miroirs chez l'observateur [65]. De plus, les aires miroirs chez l'homme s'activent non seulement à l'exécution et à l'observation d'actions, mais aussi au bruit d'une action [66-68]. L'étude de Gazzola et al. [67] s'est intéressée aux activités (fMRI) des régions associées aux actions " casser une cacahuète ", " déchirer du papier " et " manipuler entre les lèvres ", sous trois conditions : " exécution ", " observation " et " écoute ». Dans toutes les conditions, ils enregistrent une activité significative au niveau de l'IPL, BA44, BA6 et du STS. Par ailleurs, ils confirment une organisation somatotopique des actions des mains et de la bouche, liée respectivement à BA6 et à BA44 [67].

Plusieurs études montrent également que les neurones miroirs seraient sensibles à l'intention motrice présumée de l'action [69-71]. En particulier, l'expérience d'Iacoboni et al. [71] s'intéresse à l'activité des aires associées à l'action " saisir " dans trois conditions différentes : "intention », " action ", " contexte ». Dans chaque condition, deux vidéos différentes sont montrées aux sujets. La condition ("intention ") présente deux scènes où se produisent l'action « saisir une tasse » dans des contextes suggérant respectivement le début ou la fin du déjeuner. Dans le premier cas, l'ensemble 
de la scène suggère que l'action réalisée est " saisir pour boire ". Dans l'autre cas, l'action semble être " saisir pour ranger ». Dans les deux cas, la prise de mains est différente : l'une par l'anse, l'autre par le corps de la tasse. La condition (" action ") montre les mêmes actions " saisir une tasse " (par l'anse ou le corps de la tasse) en dehors de tout contexte. Enfin, la dernière condition ( " contexte ») montre les décors, sans production d'action ni présence de main. L'étude rapporte trois résultats :

- dans la condition " contexte " les aires frontales s'activent, mais pas les aires pariétales, ni le STS. D'après les auteurs, ceci est imputable à l'activité des neurones canoniques ;

- dans les conditions " intention " et " action " les aires pariétofrontales classiquement associées au RNM s'activent, y compris le STS ;

- dans la condition " intention ", la partie postérieure de l'IFG s'active significativement davantage que dans les deux autres conditions. D’après les auteurs, cette activité serait le signe physiologique de l'anticipation des actions possibles à venir, " boire " ou « ranger » $[71]$

Ces études suggèrent que l'homme possède, à l'instar du singe, un RNM impliqué dans la simulation et la reconnaissance de l'action. Cependant, outre les similitudes, une différence fondamentale apparaît entre les deux espèces. Les neurones miroirs du singe ne semblent répondre qu'à des actions. Or, nous observons chez l'homme une activité miroir pour des mouvements dénués de toute valeur sémantique [57]. En particulier, l'étude de Buccino et al. [60] montre une réponse miroir y compris à l'observation d'actions mimées. Beaucoup d'études EEG démontrant une désynchronisation du rythme mu à l'observation ont été réalisées dans le cas de mouvements globaux du corps, tels que la marche par exemple $[43,72,73]$. De plus, pour produire une réponse miroir chez le singe dans le cadre d'une observation à la $3^{\mathrm{e}}$ personne, il semble nécessaire que celui-ci voit l'ensemble du corps de l'acteur [26]. Or, l'activité miroir chez l'homme est évoquée y compris lorsque le sujet observe des mouvements isolés d'un membre [74]. Différentes études par TMS montrent également chez l'homme, une modulation de l'excitabilité corticale tout au long du mouvement observé et en fonction des différentes séquences qui le composent $[75,76]$. Une étude récente montre en outre que le rythme mu est sensible aux caractéristiques cinématiques saillantes (point d'inflexion de la trajectoire) d'un geste [77].

\section{Fonction des neurones miroirs}

\subsection{Direct-matching hypothesis}

Historiquement, le qualificatif « miroir » a été attribué aux neurones miroirs selon l'idée qu'ils sont conjointement actifs chez l'effecteur et l'observateur d'une action. Ils formeraient ainsi une activité similaire et simultanée, " en miroir ", chez les deux individus. Compte tenu de leur relation avec le but et l'intention présumés de l'action, l'équipe de recherche de l'Université de Parme, à l'origine de la découverte des neurones miroirs, suggère que ces neurones seraient impliqués dans la compréhension de l'action, à partir de la cartographie motrice de l'action observée sur le répertoire moteur de l'observateur (direct-matching hypothesis, [4]). En d'autres termes, le traitement de l'information issue de l'observation d'une action évoquerait une copie motrice de celleci au niveau des aires motrices de l'observateur. Cette hypothèse d'une copie motrice (efferent copy) a été empruntée au domaine du contrôle moteur où ce type de transfert d'information a été suggéré et démontré dans différents systèmes neuronaux. Les commandes élaborées par le cortex moteur étant par exemple transférées vers différentes régions du cerveau dont le cervelet afin d'activer un simulateur d'actions [78]. Dans le cas des neurones miroirs, cette copie interpersonnelle de l'action permettrait à l'observateur de reconnaître un événement visuel comme un mouvement du corps, de le différencier d'autres gestes analogues, de se le représenter à partir du contexte comme une action orientée vers un ou plusieurs buts, pour enfin, interpréter l'action observée à travers son propre schéma moteur et y réagir de façon appropriée.

\subsection{Comprendre ou prédire l'action?}

Compte tenu du rapport étroit entre l'activité des neurones miroirs et le but et l'intention de l'action observée, tant chez le singe que chez l'homme, de nombreux d'auteurs suggèrent que les neurones miroirs codent en eux-mêmes le but et l'intention de l'action observée. Conformément à la direct-matching hypothesis, ils postulent ainsi que le réseau de neurones miroirs est la base neurale de notre capacité à comprendre l'action d'autrui. Cette conception rencontre néanmoins de sérieuses critiques. Hickok [79] souligne en effet qu'il est tout à fait possible de comprendre une action sans jamais l'avoir réalisée. Pour reprendre son exemple : « un néophyte de la musique peut reconnaître une personne jouant du saxophone sans jamais avoir touché l'instrument, de la même façon que nous pouvons reconnaître des actions d'autres animaux (aboyer, voler) " [79]. Il y aurait ainsi une compréhension possible de l'action sans une nécessaire implication des fonctions motrices ou miroirs. D'un point de vu expérimental, l'étude de Buccino et al. [65] décrite cidessus, montre notamment que l'action intransitive " aboyer " du chien ne semble pas activer les aires miroirs de l'humain observateur, au même titre que celles réalisées par l'homme (" parler ») ou le singe (" claquer des lèvres »). Pourtant, " aboyer " n'a pas moins de valeur sémantique que " claquer des lèvres". Tous les participants ont, vraisemblablement, compris l'action " aboyer » du chien, notamment parce qu'ils peuvent la nommer. Une étude de Catmur et al. [80] montre également que les neurones miroirs peuvent changer de comportement après un entraînement sensorimoteur. Or, la compréhension du sujet vis-à-vis des actions effectuées ou observées n'est évidemment pas modifiée après l'entraînement. Il semble dès lors peu probable que les aires motrices/miroirs soient le siège de la compréhension de l'action. Comme nous l'avons vu par ailleurs, les études relatives au but et à l'intention de l'action chez le singe $[13,16]$ impliquent que celui-ci est capable de déduire le but de l'action à partir du contexte. L'étude de Livi et al. [21] chez le singe suggère en outre, que le traitement des informations liées au contexte précède et contribue à générer l'objectif de la tâche. De même, la compréhension d'un acte moteur réalisé en présence ou en absence d'obstacle implique un traitement de l'information visuelle préalable assumée par le gyrus temporal moyen et le STS dans la voie ventrale [81].

De nombreuses critiques ont ainsi mis en évidence le manque de précision quant à l'emploi du terme " comprendre " [82-84] ainsi que le manque de recule quant au rôle des neurones miroirs au sein d'un maillage d'interdépendances neurocognitif et sensoriel [85-87]. Décomposant le sens du terme " comprendre l'action ", Thompson et al. [83] soulignent notamment que les neurones miroirs contribuent essentiellement à l'identification de l'action à partir des caractéristiques élémentaires du mouvement (et non à l'identification du but de l'action ou de l'intention de l'acteur). En cause, l'identification du but et de l'intention de l'action implique un niveau de généralité (incluant toutes les actions ayant le même but ou la même intention) auquel peu de neurones miroirs semblent répondre conformément [82]. Certains auteurs suggèrent en outre que les neurones miroirs acquièrent leurs propriétés par apprentissage sensori-moteur associatif $[87,88]$. D'après ces auteurs, loin d'être la propriété première des neurones miroirs, le processus de généralisation permettant d'identifier le but et l'intention de 
l'action par l'observation serait ainsi le produit d'un apprentissage associatif visuo-moteur contingent et sensible au contexte [82].

D'après Csibra et al. [89], plutôt que de jouer le rôle d'une simulation " rétrodictive " de l'action selon une procédure " percevoir-pour-comprendre " (bottom-up), les neurones miroirs joueraient davantage le rôle d'une simulation prédictive de l'action selon une procédure " percevoir-pour-agir » (top-down). De même Kilner et al. [90] suggèrent que la fonction des neurones miroirs est de déduire l'intention des actions, selon un codage prédictif (Bayésien) du mouvement. Ces perspectives prédictives sont notamment étayées par une récente étude sur le singe démontrant que lors de l'exécution d'une action, l'activité des neurones miroirs précède généralement celle des neurones moteurs non-miroirs, suggérant un rôle particulier dans la mise en œuvre de la tâche motrice [91]. Dans une étude sur l'observation de la marche, nous avons également mis en évidence que celle-ci désynchronise le rythme mu davantage lorsque les images successives sont renversées à $180^{\circ}$ ou mélangées, qu'en condition normale. Ces données suggèrent que le rythme mu n'est pas tant impliqué dans la compréhension du mouvement que dans sa reconstruction [43]. De même, une étude récente chez le nourrisson (de 9 mois) montre une désynchronisation du rythme mu lors de l'observation d'un mouvement de préhension affiché par point lumineux, alors même que les éléments picturaux sont manquants pour reconnaître l'action [92]. Une étude de Pomiechowska et al. [93] indique en outre que la désynchronisation du rythme mu est modulée par l'interprétation de l'action observée, qui serait déduite par inférence et codée en dehors du système moteur.

\subsection{Simuler pour comprendre?}

Bien que les neurones miroirs ne soient pas en eux-mêmes le substrat de la compréhension de l'action, il est possible qu'ils y contribuent tout de même. Mahon et al. [94] postulent notamment que la "compréhension " implique un niveau abstrait de représentation (qu'ils nomment " concepts ») distinct des systèmes sensorimoteurs. D'après ces auteurs, ces concepts suffisent à la " compréhension » mais peuvent être associés à des représentations sensorimotrices qui les enrichissent en leur fournissant un aspect sensitif et contextuel [94]. Ainsi, comme nous l'avons vu, un néophyte de la musique pourrait avoir un concept parfaitement viable de "jouer du saxophone ", sans jamais avoir touché l'instrument [79]. Son concept ne serait pas lié aux informations motrices de l'action. Néanmoins, savoir comment tenir le saxophone et placer les doigts pour jouer, pourrait enrichir son concept abstrait en lui offrant une association au système sensorimoteur. De plus, cette association pourrait l'amener à une " compréhension » étendue de " jouer du saxophone ». Par exemple, à la vue d'un saxophoniste qui tient mal son instrument, l'observateur pourrait comprendre que le joueur n'est pas un expert [79]. Ainsi, d'après ce modèle, il est probable que les concepts émergent d'un processus d'association de nombreuses aires corticales, qui en donneraient chacune une composante. La « compréhension » émergerait alors d'un processus de congruence entre les différentes composantes de l'action observée. Elle serait en outre actée par la confirmation à posteriori de l'interprétation de l'action par les faits observés.

Dans ce contexte associatif, il semble que ce soit la composante sensorimotrice que Rizzolatti et al. [95,96] nomment " understanding from inside ", qui pourrait être assurée par les neurones miroirs. À ce titre, une étude de notre équipe indique que, outre les commandes motrices, le RNM intègre les entrées somatosensorielles associées à l'observation du mouvement d'un geste moteur [97]. Cebolla et al. [97] ont en effet étudié le potentiel somesthésique évoqué par la stimulation du nerf médian au poignet lors de l'observation d'un mouvement de la main. Les résultats de l'étude montrent que l'amplitude de l'onde N30 est augmentée lors de l'observation du mouvement. En outre, celle-ci s'accompagne d'une augmentation de la puissance et de la mise en phase des oscillations alpha au niveau de la région précentrale (rythme mu) et serait expliquée par la contribution d'aires miroirs (en particulier du gyrus angulaire). Corroborant l'étude de Coll et al. [53], ces résultats suggèrent que le RNM code non seulement les aspects complexes de la commande motrice mais intègre également les réafférences sensorielles (cutanées et proprioceptives).

L'hypothèse d'une contribution des neurones miroirs à la compréhension est corroborée par plusieurs études suggérant que ces neurones jouent un rôle actif lors de l'observation de l'action [98-102]. Notamment, Cole et al. [102] ont mis en évidence que l'activité cortico-spinale évoquée par TMS est plus importante lorsqu'un sujet observe des actions dans le but d'inférer l'intention de celles-ci. De même, l'étude récente d'Ikeda et al. [99] compare la désynchronisation du rythme mu dans trois conditions d'observation :

- observer dans le but de comprendre l'intention de l'action observée ;

- observer dans le but d'imiter de manière imaginaire l'action observée

- observer sans intention particulière.

Les auteurs mettent en évidence une désynchronisation du rythme mu plus importante lorsque le sujet observe un acte moteur avec le but de comprendre l'intention de l'action observée, comparativement aux deux autres conditions [99]. En outre, plusieurs études suggèrent qu'en dehors de toute information contextuelle, les neurones miroirs permettent de déduire l'intention d'une action à partir de faibles variations dans la cinématique des mouvements $[100,101]$.

\section{Conclusion}

Les auteurs à l'origine de la découverte des neurones dits " miroirs » chez le singe ont proposé, sur la base de la direct-matching hypothesis, un modèle de la compréhension de l'action dans lequel ces neurones seraient l'élément central. Fidèle à cette conception bottom-up, ils l'ont étendue, chez l'homme, à un modèle miroir de la compréhension de l'autre au sens large, en soutenant que le mécanisme miroir serait à la base de l'imitation, de l'empathie et du langage $[4,103]$. De nombreuses études ont néanmoins nuancé le modèle, mettant en évidence l'importance du contexte dans la compréhension de l'action. Sans réduire l'importance de ces neurones dans la cognition, ces critiques tentent de démystifier les neurones " miroirs " en les replaçant au sein d'un maillage d'interdépendances neurocognitives [84,85,87]. En somme, la caractéristique essentielle de ces neurones est d'associer le traitement de l'information visuelle avec celui de l'information motrice. Leur fonction serait ainsi de fournir une simulation motrice de l'action observée, à partir des informations visuelles élémentaires de celle-ci. Ils peuvent contribuer de concert avec d'autres aires cérébrales non-miroirs, à l'identification/prédiction de but de l'action et à l'interprétation de l'intention de l'acteur qui l'effectue. Ils pourraient en outre, enrichir la compréhension du sujet à propos de l'action observée au travers des aspects moteurs de celle-ci.

À ce stade, il nous semble que l'exploration des relations entre l'information visuelle liée au contexte et l'activité miroir mériterait d'être approfondie. En particulier, l'étude des composantes temporelles de l'activité des neurones miroirs, dans le cadre de l'observation d'action où le contexte entre en contradiction avec le but de l'action, permettrait probablement de préciser les relations fonctionnelles entre les différentes composantes de la compréhension de l'action (simuler vs interpréter vs prédire vs 
comprendre). À l'instar de l'étude fMRI de Jastroff et al. [81], une étude de l'influence du contexte sur la désynchronisation du rythme mu lors de l'observation de l'action nous semble manquer aujourd'hui. L'étude de la connectivité et des synchronisations des différentes aires cérébrales en jeu lors de l'observation d'une action apporterait probablement des informations importantes quant à la contribution dynamique du RNM à la compréhension de l'action d'autrui. En outre, les données futures issues de l'enregistrement simultané des unités neuronales isolées, des MUA, des LFP, de l'intracérébral stereo-EEG [104,105] et de l'EEG de surface devraient nous permettent de mieux comprendre les fondements oscillatoires sous-jacents à l'activité du RNM.

Pour conclure, il nous paraît crucial que les futures études définissent de manière explicite et précise les termes et les processus relatifs à ce qui est communément nommé la " compréhension " de l'action. Compte tenu en outre du développement important de la littérature relative aux neurones miroirs, une compréhension précise des propriétés et des fonctions présumées de ces neurones paraît primordiale pour éviter les simplifications abusives. Par ailleurs, le développement de nouvelles approches expérimentales tant chez l'animal que chez l'homme, devrait permettre de renforcer les différents modèles relatifs aux phénomènes complexes qui impliquent les neurones miroirs, telle que l'imitation [106,107], l'empathie [108], la coordination sociale [109-111] ou encore l'autisme [112].

\section{Déclaration de liens d'intérêts}

Les auteurs déclarent ne pas avoir de liens d'intérêts.

\section{Remerciements}

Nous remercions chaleureusement M. Petieau, E. Pecoraro, E. Hortmans, T. D'Angelo et E. Toussaint pour leur expertise technique. Ce travail a été financé par l'Université Libre de Bruxelles (Belgique), le Fonds G. Leibu et la Fondation Brain Society.

\section{Références}

[1] di Pellegrino G, Fadiga L, Fogassi L, et al. Understanding motor events: a neurophysiological study. Exp Brain Res 1992;91:176-80, http://dx.doi.org/10.1007/BF00230027.

[2] Gallese V, Fadiga L, Fogassi L, et al. Action recognition in the premotor cortex. Brain J Neurol 1996;119(Pt 2):593-609, http://dx.doi.org/10.1093/brain/119.2.593.

[3] Rizzolatti G, Fadiga L, Gallese V, et al. Premotor cortex and the recognition of motor actions. Brain Res Cogn Brain Res 1996;3:131-41, http://dx.doi.org/10.1016/0926-6410(95)00038-0.

[4] Rizzolatti G, Craighero L. The mirror-neuron $\begin{array}{llll}\text { system. } & \text { Annu } & \text { Rev } & \text { Neurosci } \\ \text { 2004;27:169-92, }\end{array}$ http://dx.doi.org/10.1146/annurev.neuro.27.070203.144230.

[5] Ferrari PF, Gallese V, Rizzolatti G, et al. Mirror neurons responding to the observation of ingestive and communicative mouth actions in the monkey ventral premotor cortex. Eur J Neurosci 2003;17:1703-14, http://dx.doi.org/10.1046/j.1460-9568.2003.02601.x.

[6] Cisek P, Kalaska JF. Neural correlates of mental rehearsal in dorsal premotor cortex. Nature 2004;431:993-6, http://dx.doi.org/10.1038/nature03005.

[7] Tkach D, Reimer J, Hatsopoulos NG. Congruent activity during action and action observation in motor cortex. J Neurosci 2007;27:13241-50, http://dx.doi.org/10.1523/JNEUROSCI.2895-07.2007.

[8] Bonini L, Maranesi M, Livi A, et al. Space-dependent representation of objects and other's action in monkey ventral premotor grasping neurons. J Neurosci Off J Soc Neurosci 2014;34:4108-19, http://dx.doi.org/10.1523/JNEUROSCI.4187-13.2014.

[9] Caggiano V, Pomper JK, Fleischer F, et al. Mirror neurons in monkey area F5 do not adapt to the observation of repeated actions. Nat Commun 2013;4:1433, http://dx.doi.org/10.1038/ncomms2419.

[10] Kilner JM, Kraskov A, Lemon RN. Do monkey F5 mirror neurons show changes in firing rate during repeated observation of natural actions? J Neurophysiol 2014;111:1214-26, http://dx.doi.org/10.1152/jn.01102.2012.

[11] Papadourakis V, Raos V. Evidence for the representation of movement kinematics in the discharge of F5 mirror neurons during the observation of transitive and intransitive actions. J Neurophysiol 2017;118:3215-29, http://dx.doi.org/10.1152/jn.00816.2016.
[12] Rochat MJ, Caruana F, Jezzini A, et al. Responses of mirror neurons in area F5 to hand and tool grasping observation. Exp Brain Res 2010;204:605-16, http://dx.doi.org/10.1007/s00221-010-2329-9.

[13] Umiltà MA, Kohler E, Gallese V, et al. I know what you are doing. A neurophysiological study. Neuron 2001;31:155-65, http://dx.doi.org/10.1016/s0896-6273(01)00337-3.

[14] Kohler E, Keysers C, Umiltà MA, et al. Hearing sounds, understanding actions: action representation in mirror neurons. Science 2002;297:846-8, http://dx.doi.org/10.1126/science.1070311.

[15] Keysers C, Kohler E, Umiltà MA, et al. Audiovisual mirror neurons and action recognition. Exp Brain Res 2003;153:628-36, http://dx.doi.org/10.1007/s00221-003-1603-5.

[16] Fogassi L, Ferrari PF, Gesierich B, et al. Parietal lobe: from action organization to intention understanding. Science 2005;308:662-7. http://dx.doi.org/10.1126/science.1106138.

[17] Coudé G, Festante F, Cilia A, et al. Mirror neurons of ventral premotor cortex are modulated by social cues provided by others' gaze. J Neurosci Off J Soc Neurosci 2016;36:3145-56, http://dx.doi.org/10.1523/JNEUROSCI.3220-15.2016.

[18] Murata A, Gallese V, Luppino G, et al. Selectivity for the shape, size, and orientation of objects for grasping in neurons of monkey parietal area AIP. J Neurophysiol 2000;83:2580-601, http://dx.doi.org/10.1152/jn.2000.83.5. 2580.

[19] Lanzilotto M, Maranesi M, Livi A, et al. Stable readout of observed actions from format-dependent activity of monkey's anterior intraparietal neurons. Proc Natl Acad Sci U S A 2020;117:16596-605, http://dx.doi.org/10.1073/pnas.2007018117.

[20] Bonini L, Rozzi S, Serventi FU, et al. Ventral premotor and inferior parietal cortices make distinct contribution to action organization and intention understanding. Cereb Cortex N Y N 1991 2010;20:1372-85, http://dx.doi.org/10.1093/cercor/bhp200.

[21] Livi A, Lanzilotto M, Maranesi M, et al. Agent-based representations of objects and actions in the monkey pre-supplementary motor area. Proc Natl Acad Sci U S A 2019;116:2691-700, http://dx.doi.org/10.1073/pnas.1810890116.

[22] Dushanova J, Donoghue J. Neurons in primary motor cortex engaged during action observation. Eur J Neurosci 2010;31:386-98, http://dx.doi.org/10.1111/j.1460-9568.2009.07067.x.

[23] Bruni S, Gerbella M, Bonini L, et al. Cortical and subcortical connections of parietal and premotor nodes of the monkey hand mirror neuron network. Brain Struct Funct 2018;223:1713-29, http://dx.doi.org/10.1007/s00429-017-1582-0.

[24] Petrides M, Pandya DN. Projections to the frontal cortex from the posterior parietal region in the rhesus monkey. J Comp Neurol 1984;228:105-16, http://dx.doi.org/10.1002/cne.902280110.

[25] Matelli M, Camarda R, Glickstein M, et al. Afferent and efferent projections of the inferior area 6 in the macaque monkey. J Comp Neurol 1986;251:281-98, http://dx.doi.org/10.1002/cne.902510302.

[26] Nelissen K, Luppino G, Vanduffel W, et al. Observing others: multiple action representation in the frontal lobe. Science 2005;310:332-6, http://dx.doi.org/10.1126/science.1115593.

[27] Nelissen K, Borra E, Gerbella $M$, et al. Action observation circuits in the macaque monkey cortex. J Neurosci 2011;31:3743-56, http://dx.doi.org/10.1523/JNEUROSCI.4803-10.2011.

[28] Caggiano V, Fogassi L, Rizzolatti G, et al. Mirror neurons encode the subjective value of an observed action. Proc Natl Acad Sci U S A 2012;109:11848-53, http://dx.doi.org/10.1073/pnas.1205553109.

[29] Maranesi M, Livi A, Bonini L. Spatial and viewpoint selectivity for others' observed actions in monkey ventral premotor mirror neurons. Sci Rep 2017;7:8231, http://dx.doi.org/10.1038/s41598-017-08956-1.

[30] Caggiano V, Fogassi L, Rizzolatti G, et al. Mirror neurons differentially encode the peripersonal and extrapersonal space of monkeys. Science 2009;324:403-6, http://dx.doi.org/10.1126/science.1166818.

[31] Caggiano V, Fogassi L, Rizzolatti G, et al. View-based encoding of actions in mirror neurons of area $\mathrm{f} 5$ in macaque premotor cortex. Curr Biol CB 2011;21:144-8, http://dx.doi.org/10.1016/j.cub.2010.12.022.

[32] Rizzolatti G, Luppino G. The cortical motor system. Neuron 2001;31:889-901, http://dx.doi.org/10.1016/S0896-6273(01)00423-8.

[33] Kraskov A, Dancause N, Quallo MM, et al. Corticospinal neurons in macaque ventral premotor cortex with mirror properties: a potential mechanism for action suppression? Neuron 2009;64:922-30, http://dx.doi.org/10.1016/j.neuron.2009.12.010.

[34] Kraskov A, Philipp R, Waldert S, et al. Corticospinal mirror neurons. Philos Trans R Soc Lond B Biol Sci 2014;369:20130174, http://dx.doi.org/10.1098/rstb.2013.0174.

[35] Vigneswaran G, Philipp R, Lemon RN, et al. M1 corticospinal mirror neurons and their role in movement suppression during action observation. Curr Biol CB 2013;23:236-43, http://dx.doi.org/10.1016/j.cub.2012.12.006.

[36] Jerjian SJ, Sahani M, Kraskov A. Movement initiation and grasp representation in premotor and primary motor cortex mirror neurons. ELife 2020:9, http://dx.doi.org/10.7554/eLife.54139.

[37] Faure J, Cohen-Seat G. Responses to sensory stimulation of activation induced by projection of a film. Rev Neurol (Paris) 1954;90:307-11.

[38] Cochin S, Barthelemy C, Roux S, et al. Observation and execution of movement: similarities demonstrated by quantified electroencephalography. Eur J Neurosci 1999;11:1839-42, http://dx.doi.org/10.1046/j.1460-9568.1999.00598.x. 
[39] Babiloni C, Babiloni F, Carducci F, et al. Human cortical electroencephalography (EEG) rhythms during the observation of simple aimless movements: a high-resolution EEG study. NeuroImage 2002;17:559-72.

[40] Pineda JA. The functional significance of mu rhythms: translating "seeing" and "hearing" into "doing.". Brain Res Brain Res Rev 2005;50:57-68, http://dx.doi.org/10.1016/j.brainresrev.2005.04.005.

[41] Hari R. Action-perception connection and the cortical mu rhythm. Prog Brain Res 2006;159:253-60, http://dx.doi.org/10.1016/S0079-6123(06)59017-X.

[42] Perry A, Bentin S. Mirror activity in the human brain while observing hand movements: a comparison between EEG desynchronization in the $\mu$-range and previous fMRI results. Brain Res 2009;1282:126-32, http://dx.doi.org/10.1016/j.brainres.2009.05.059.

[43] Zarka D, Cevallos C, Petieau M, et al. Neural rhythmic symphony of human walking observation: upside-down and uncoordinated condition on cortical theta, alpha, beta and gamma oscillations. Front Syst Neurosci 2014;8:169, http://dx.doi.org/10.3389/fnsys.2014.00169.

[44] Fox NA, Bakermans-Kranenburg MJ, Yoo KH, et al. Assessing human mirror activity with EEG mu rhythm: A meta-analysis. Psychol Bull 2016;142:291-313, http://dx.doi.org/10.1037/bul0000031.

[45] Arnstein D, Cui F, Keysers C, et al. $\mu$-suppression during action observation and execution correlates with BOLD in dorsal premotor, inferior parietal, and SI cortices. J Neurosci Off J Soc Neurosci 2011;31:14243-9, http://dx.doi.org/10.1523/JNEUROSCI.0963-11.2011.

[46] Braadbaart L, Williams JHG, Waiter GD. Do mirror neuron areas mediate mu rhythm suppression during imitation and action observation? Int Organ Psychophysiol 2013;89:99-105, http://dx.doi.org/10.1016/j.ijpsycho.2013.05.019.

[47] Urgen BA, Plank M, Ishiguro $\mathrm{H}$, et al. EEG theta and Mu oscillations during perception of human and robot actions. Front Neurorobotics 2013;7:19, http://dx.doi.org/10.3389/fnbot.2013.00019.

[48] Debnath R, Salo VC, Buzzell GA, et al. Mu rhythm desynchronization is specific to action execution and observation: Evidence from time-frequency and connectivity analysis. NeuroImage 2019;184:496-507, http://dx.doi.org/10.1016/j.neuroimage.2018.09.053.

[49] Prinsen J, Alaerts K. Enhanced mirroring upon mutual gaze: multimodal evidence from TMS-assessed corticospinal excitability and the EEG mu rhythm. Sci Rep 2020;10:20449, http://dx.doi.org/10.1038/s41598-02077508-X.

[50] van Elk M, van Schie HT, Hunnius S, et al. You'll never crawl alone: neurophysiological evidence for experience-dependent motor resonance in infancy. Neurolmage 2008;43:808-14, http://dx.doi.org/10.1016/j.neuroimage.2008.07.057.

[51] Brunsdon VEA, Bradford EEF, Smith L, et al. Short-term physical training enhances mirror system activation to action observation. Soc Neurosci 2020;15:98-107, http://dx.doi.org/10.1080/17470919.2019.1660708.

[52] Cannon EN, Yoo KH, Vanderwert RE, et al. Action experience, more than observation, influences mu rhythm desynchronization. PloS One 2014;9:e92002, http://dx.doi.org/10.1371/journal.pone.0092002.

[53] Coll M-P, Press C, Hobson H, et al. Crossmodal classification of mu rhythm activity during action observation and execution suggests specificity to somatosensory features of actions. J Neurosci Off J Soc Neurosci 2017;37:5936-47, http://dx.doi.org/10.1523/JNEUROSCI.3393-16.2017.

[54] Bimbi M, Festante F, Coudé G, et al. Simultaneous scalp recorded EEG and local field potentials from monkey ventral premotor cortex during action observation and execution reveals the contribution of mirror and motor neurons to the mu-rhythm. Neurolmage 2018;175:22-31, http://dx.doi.org/10.1016/j.neuroimage.2018.03.037.

[55] Rizzolatti G, Fadiga L, Matelli M, et al. Localization of grasp representations in humans by PET: 1. Observation versus execution. Exp Brain Res 1996;111:246-52, http://dx.doi.org/10.1007/BF00227301.

[56] Grafton ST, Arbib MA, Fadiga L, et al. Localization of grasp representations in humans by positron emission tomography. Exp Brain Res 1996;112:103-11, http://dx.doi.org/10.1007/BF00227183.

[57] Decety J, Grèzes J, Costes N, et al. Brain activity during observation of actions. Influence of action content and subject's strategy. Brain 1997;120:1763-77, http://dx.doi.org/10.1093/brain/120.10.1763.

[58] Nishitani N, Hari R. Temporal dynamics of cortical representation for action. Proc Natl Acad Sci U S A 2000;97:913-8, http://dx.doi.org/10.1073/pnas.97.2.913.

[59] Molenberghs P, Cunnington R, Mattingley JB. Brain regions with mirror properties: a meta-analysis of 125 human fMRI studies. Neurosci Biobehav Rev 2012;36:341-9, http://dx.doi.org/10.1016/j.neubiorev.2011.07.004.

[60] Buccino G, Binkofski F, Fink GR, et al. Action observation activates premotor and parietal areas in a somatotopic manner: an fMRI study. Eur J Neurosci $2001 ; 13: 400-4$

[61] Jastorff J, Begliomini C, Fabbri-Destro M, et al. Coding observed motor acts: different organizational principles in the parietal and premotor cortex of humans. J Neurophysiol 2010;104:128-40, http://dx.doi.org/10.1152/jn.00254.2010.

[62] Simon S, Mukamel R. Sensitivity to perception level differentiates two subnetworks within the mirror neuron system. Soc Cogn Affect Neurosci 2017;12:861-70, http://dx.doi.org/10.1093/scan/nsx015.

[63] Mountcastle VB. Modality and topographic properties of single neurons of cat's somatic sensory cortex. J Neurophysiol 1957;20:408-34, http://dx.doi.org/10.1152/jn.1957.20.4.408.
[64] Mukamel R, Ekstrom AD, Kaplan J, et al. Single-neuron responses in humans during execution and observation of actions. Curr Biol CB 2010;20:750-6, http://dx.doi.org/10.1016/j.cub.2010.02.045.

[65] Buccino G, Lui F, Canessa N, et al. Neural circuits involved in the recognition of actions performed by nonconspecifics: an FMRI study. J Cogn Neurosci 2004;16:114-26, http://dx.doi.org/10.1162/089892904322755601.

[66] Aziz-Zadeh L, Iacoboni M, Zaidel E, et al. Left hemisphere motor facilitation in response to manual action sounds. Eur J Neurosci 2004;19:2609-12. http://dx.doi.org/10.1111/j.0953-816X.2004.03348.x.

[67] Gazzola V, Aziz-Zadeh L, Keysers C. Empathy and the somatotopic auditory mirror system in humans. Curr Biol CB 2006;16:1824-9, http://dx.doi.org/10.1016/j.cub.2006.07.072.

[68] Lepage J-F, Tremblay S, Nguyen DK, et al. Action related sounds induce early and late modulations of motor cortex activity. Neuroreport 2010;21:250-3, http://dx.doi.org/10.1097/WNR.0b013e328334ddcc.

[69] Schubotz RI, von Cramon DY. Functional organization of the lateral premotor cortex: fMRI reveals different regions activated by anticipation of object properties, location and speed. Brain Res Cogn Brain Res 2001;11:97-112, http://dx.doi.org/10.1016/s0926-6410(00)00069-0.

[70] Schubotz RI, von Cramon DY. Predicting perceptual events activates corresponding motor schemes in lateral premotor cortex: an fMRI study. Neurolmage 2002;15:787-96, http://dx.doi.org/10.1006/nimg.2001.1043.

[71] Iacoboni M, Molnar-Szakacs I, Gallese V, et al. Grasping the intentions of others with one's own mirror neuron system. PLoS Biol 2005;3:e79, http://dx.doi.org/10.1371/journal.pbio.0030079.

[72] Servos P, Osu R, Santi A, et al. The neural substrates of biological motion perception: an fMRI study. Cereb Cortex N Y N 1991 2002;12:772-82, http://dx.doi.org/10.1093/cercor/12.7.772.

[73] Ulloa ER, Pineda JA. Recognition of point-light biological motion: mu rhythms and mirror neuron activity. Behav Brain Res 2007;183:188-94, http://dx.doi.org/10.1016/j.bbr.2007.06.007.

[74] Turella L, Pierno AC, Tubaldi F, et al. Mirror neurons in humans: consisting or confounding evidence? Brain Lang 2009;108:10-21, http://dx.doi.org/10.1016/j.bandl.2007.11.002.

[75] Gangitano M, Mottaghy FM, Pascual-Leone A. Phase-specific modulation of cortical motor output during movement observation. Neuroreport 2001;12:1489-92, http://dx.doi.org/10.1097/00001756-200105250-00038.

[76] Borroni P, Baldissera F. Activation of motor pathways during observation and execution of hand movements. Soc Neurosci 2008;3:276-88, http://dx.doi.org/10.1080/17470910701515269.

[77] Cabrera ME, Novak K, Foti D, et al. Electrophysiological indicators of gesture perception. Exp Brain Res 2020;238:537-50, http://dx.doi.org/10.1007/s00221-020-05724-y.

[78] Cheron G, Dan B, Márquez-Ruiz J. Translational approach to behavioral learning: lessons from cerebellar plasticity. Neural Plast 2013;2013:853654, http://dx.doi.org/10.1155/2013/853654.

[79] Hickok G. Eight problems for the mirror neuron theory of action understanding in monkeys and humans. J Cogn Neurosci 2009;21:1229-43, http://dx.doi.org/10.1162/jocn.2009.21189.

[80] Catmur C, Walsh V, Heyes C. Sensorimotor learning configures the human mirror system. Curr Biol CB 2007;17:1527-31, http://dx.doi.org/10.1016/j.cub.2007.08.006.

[81] Jastorff J, Clavagnier S, Gergely G, et al. Neural mechanisms of understanding rational actions: middle temporal gyrus activation by contextual violation. Cereb Cortex N Y N 1991 2011;21:318-29, http://dx.doi.org/10.1093/cercor/bhq098.

[82] Cook R, Bird G. Do mirror neurons really mirror and do they really code for action goals? Cortex J Devoted Study Nerv Syst Behav 2013;49:2944-5, http://dx.doi.org/10.1016/j.cortex.2013.05.006.

[83] Thompson EL, Bird G, Catmur C. Conceptualizing and testing action understanding. Neurosci Biobehav Rev 2019;105:106-14, http://dx.doi.org/10.1016/j.neubiorev.2019.08.002.

[84] Hickok G. Do mirror neurons subserve action understanding? Neurosci Lett 2013;540:56-8, http://dx.doi.org/10.1016/j.neulet.2012.11.001.

[85] Cook R, Bird G, Catmur C, et al. Mirror neurons: from origin to function. Behav Brain Sci 2014;37:177-92, http://dx.doi.org/10.1017/S0140525X13000903.

[86] Pascolo PB, Bucci A. Mirror neurons in the macaque monkey: a critical review. Crit Rev Biomed Eng 2019;47:507-13, http://dx.doi.org/10.1615/CritRevBiomedEng.2020031420.

[87] Heyes C. Where do mirror neurons come from? Neurosci Biobehav Rev 2010;34:575-83, http://dx.doi.org/10.1016/j.neubiorev.2009.11.007.

[88] Heyes C. A new approach to mirror neurons: developmental history, systemlevel theory and intervention experiments. Cortex J Devoted Study Nerv Syst Behav 2013;49:2946-8, http://dx.doi.org/10.1016/j.cortex.2013.07.002.

[89] Csibra G, Gergely G. "Obsessed with goals": functions and mechanisms of teleological interpretation of actions in humans. Acta Psychol (Amst) 2007;124:60-78, http://dx.doi.org/10.1016/j.actpsy.2006.09.007.

[90] Kilner JM, Friston KJ, Frith CD. Predictive coding: an account of the mirror neuron system. Cogn Process 2007;8:159-66, http://dx.doi.org/10.1007/s10339-007-0170-2.

[91] Mazurek KA, Schieber MH. Mirror neurons precede non-mirror neurons during action execution. J Neurophysiol 2019;122:2630-5 http://dx.doi.org/10.1152/jn.00653.2019.

[92] Quadrelli E, Roberti E, Turati C, et al. Observation of the point-light animation of a grasping hand activates sensorimotor cortex in nine-month 
-old infants. Cortex J Devoted Study Nerv Syst Behav 2019;119:373-85, http://dx.doi.org/10.1016/j.cortex.2019.07.006.

[93] Pomiechowska B, Csibra G. Motor activation during action perception depends on action interpretation. Neuropsychologia 2017;105:84-91, http://dx.doi.org/10.1016/j.neuropsychologia.2017.01.032.

[94] Mahon BZ, Caramazza A. A critical look at the embodied cognition hypothesis and a new proposal for grounding conceptual content. J Physiol-Paris 2008;102:59-70, http://dx.doi.org/10.1016/j.jphysparis.2008.03.004.

[95] Gallese V. Embodied Simulation. Its bearing on aesthetic experience and the dialogue between neuroscience and the humanities. Gestalt Theory 2019;41:113-27, http://dx.doi.org/10.2478/gth-2019-0013.

[96] Rizzolatti G, Sinigaglia C. The functional role of the parieto-frontal mirror circuit: interpretations and misinterpretations. Nat Rev Neurosci 2010;11:264-74, http://dx.doi.org/10.1038/nrn2805.

[97] Cebolla AM, Palmero-Soler E, Dan B, et al. Modulation of the N30 generators of the somatosensory evoked potentials by the mirror neuron system. NeuroImage 2014;95:48-60, http://dx.doi.org/10.1016/j.neuroimage.2014.03.039.

[98] Molenberghs P, Hayward L, Mattingley JB, et al. Activation patterns during action observation are modulated by context in mirror system areas. NeuroImage 2012;59:608-15, http://dx.doi.org/10.1016/j.neuroimage.2011.07.080.

[99] Ikeda Y, Nishimura Y, Higuchi S. Effects of the differences in mental states on the mirror system activities when observing hand actions. J Physiol Anthropol 2019;38:1, http://dx.doi.org/10.1186/s40101-018-0192-8.

[100] Cavallo A, Koul A, Ansuini C, et al. Decoding intentions from movement kinematics. Sci Rep 2016;6:37036, http://dx.doi.org/10.1038/srep37036.

[101] Koul A, Cavallo A, Cauda F, et al. Action observation areas represent intentions from subtle kinematic features. Cereb Cortex N Y N 1991 2018;28:2647-54, http://dx.doi.org/10.1093/cercor/bhy098.

[102] Cole EJ, Barraclough NE. Timing of mirror system activation when inferring the intentions of others. Brain Res 2018;1700:109-17, http://dx.doi.org/10.1016/j.brainres.2018.07.015.
[103] Rizzolatti G, Sinigaglia C. The mirror mechanism: a basic principle of brain function. Nat Rev Neurosci 2016;17:757-65, http://dx.doi.org/10.1038/nrn.2016.135.

[104] Avanzini P, Pelliccia V, Lo Russo G, et al. Multiple time courses of somatosensory responses in human cortex. NeuroImage 2018;169:212-26, http://dx.doi.org/10.1016/j.neuroimage.2017.12.037.

[105] Rizzolatti G, Fabbri-Destro M, Caruana F, et al. System neuroscience: past, present, and future. CNS Neurosci Ther 2018;24:685-93. http://dx.doi.org/10.1111/cns.12997.

[106] Cross KA, Iacoboni M. Neural systems for preparatory control of imitation. Philos Trans R Soc Lond B Biol Sci 2014;369:20130176, http://dx.doi.org/10.1098/rstb.2013.0176.

[107] Catmur C, Heyes C. Mirroring "meaningful" actions: Sensorimotor learning modulates imitation of goal-directed actions. Q J Exp Psychol 2006 2019;72:322-34, http://dx.doi.org/10.1080/17470218.2017.1344257.

[108] Bekkali S, Youssef GJ, Donaldson PH, et al. Is the putative mirror neuron system associated with empathy? A systematic review and meta-analysis. Neuropsychol Rev 2020, http://dx.doi.org/10.1007/s11065-020-09452-6.

[109] Silas J, Levy JP, Holmes A. Sensitivity of "mu" rhythm modulation to the relevance of an observed movement but not to goal congruency. Int J Psychophysiol 2012;85:168-73, http://dx.doi.org/10.1016/j.ijpsycho.2012.05.008.

[110] Yin J, Ding $X, X u ~ H$, et al. Social coordination information in dynamic chase modulates EEG mu rhythm. Sci Rep 2017;7:4782, http://dx.doi.org/10.1038/s41598-017-04129-2.

[111] Jamali M, Grannan BL, Fedorenko E, et al. Single-neuronal predictions of others' beliefs in humans. Nature 2021:1-5, http://dx.doi.org/10.1038/s41586-021-03184-0.

[112] Chan MMY, Han YMY. Differential mirror neuron system (MNS) activation during action observation with and without social-emotional components in autism: a meta-analysis of neuroimaging studies. Mol Autism 2020;11:72, http://dx.doi.org/10.1186/s13229-020-00374-x. 